\title{
Awareness, treatment, control, and determinants of dyslipidemia among adults in China: results from the China National Stroke Screening and Prevention Project (CNSSPP)
}

Sampson Opoku ( $\sim$ samblessvissions@gmail.com )

Huazhong University of Science and Technology

\section{Yong Gan}

Huazhong University of Science and Technology

\section{Emmanuel Addo-Yobo}

State University of New York Upstate Medical University

Wei Yue

Tianjin Huanhu Hospital

\section{Zhihong Wang}

shenzhen university

\section{Zuxun Lu}

Huazhong University of Science and Technology

\section{Research}

Keywords: Dyslipidemia, Determinants, Awareness, Treatment, Contr

Posted Date: June 29th, 2020

DOI: https://doi.org/10.21203/rs.3.rs-37985/v1

License: (c) (1) This work is licensed under a Creative Commons Attribution 4.0 International License. Read Full License 


\section{Abstract}

\section{Background:}

Effective management of dyslipidemia could play a vital role in the control of cardiovascular diseases. This study aimed to determine the awareness, treatment, control, and determinants of dyslipidemia in middle-aged and older Chinese adults in China.

\section{METHODS}

Using data from the 2015 CNSSPP, a nationally representative sample of 135,403 Chinese adults aged over 40 years were included in the cross-sectional analysis. Dyslipidemia was defined according to the 2016 Chinese guidelines for the management of dyslipidemia in adults. Models were constructed to adjust for sociodemographic, clinical and behavioral characteristics with bivariate and multivariable logistic regressions.

\section{RESULTS}

Overall, $51.1 \%$ of the subjects were women. Sixty-four percent $(36,958 / 57760)$ were aware of their condition, of whom $18.9 \%(6993 / 36958)$ received treatment, and of whom $7.3 \%$ (504 / 6993) had controlled lipids. Women versus men had higher proportions of awareness, treatment, and control $(69.2 \%$ vs. $58.3 \%, 63.8 \%$ vs. $64.1 \%$ and $7.7 \%$ vs.7.0\%; $p<$ $0.001)$, respectively. Dyslipidemia treatment was higher in men from rural than urban areas $(15.4 \%$ vs. $10.6 \%$; $p<0.001)$. Most subjects $(94.3 \%)$, had dyslipidemia in their family. The proportion of awareness was higher among urban compared to rural residents across all the socioeconomic regions: low-income (68.8\% vs. $60.4 \%)$, middleincome (69.4\% vs.63.9\%), and high-income (66\% vs.58.6\%), respectively (all $p<0.001)$. In the multivariable analysis, significant associations were found between awareness and women, urban residents, having a family history, and central obesity. Being a woman, having a personal history of stroke or CHD, being a drinker, having health insurance, and persons who lived in high-income regions had higher odds of receiving treatment. Adults in the $50-59$ years age group, having health insurance, central and general obesity, living in a stroke belt or northern zone, urban area, and women showed statistically significant relationships with control.

\section{CONCLUSIONS}

Dyslipidemia awareness was moderately high, but treatment and control were low. Improvements in education programs and therapeutic management may be necessary to promote and optimize the management of dyslipidemia.

\section{Introduction}

Cardiovascular diseases (CVDs) are the leading cause of morbidity and mortality globally. ${ }^{1}$ Dyslipidemia is an established risk factor for CVDs' morbidity and mortality. ${ }^{2}$ Annually, 4.4 million deaths occur worldwide as a result of raised cholesterols. ${ }^{3-5}$ Dyslipidemia awareness, treatment and control have been studied in general populations and the treatments of lipid disorders have proven to be effective in both primary and secondary prevention of cardiovascular events. ${ }^{6}$ Nevertheless, dyslipidemia screening and treatment rates have been low in low- and middleincome countries. ${ }^{5}$

With the rapid economic and health transitions coupled with lifestyle changes, dyslipidemia prevalence in China have increased recent years. The China National Diabetes and Metabolic Disorders Study reports that the levels of TC, 
LDL-C, and TG have substantially increased in recent decades, however, awareness and treatment levels still remained low. ${ }^{7}$ Song et al., in 2011 reported the awareness, treatment and control rates among dyslipidemic adults' aged $\geq 45$ years in China as $20.27 \%, 14.41 \%$ and $4.94 \%$, respectively. ${ }^{8}$ In 2014 a systematic review of observational studies on dyslipidemia in China with 387,825 subjects showed $42 \%$ prevalence rate, $24.4 \%$ awareness rate, $8.8 \%$ treatment rate, and $4.3 \%$ control rate. ${ }^{9}$ In view of the above stated studies, and with reference to the "rule of halves" framework, it can be observed that the rate of dyslipidemia awareness, treatment and control in China have been quite low. The 'rule of halves' is a proxy framework used to estimate indicators of unmet needs for chronic diseases and it stipulates that: (1) about $50 \%$ of all diseases should be diagnosed, (2) of whom about $50 \%$ should receive treatment and (3) of whom about 50\% achieve should treatment targets. ${ }^{10}$ Medication adherence and lifestyle changes are known to decrease the cost related to cardiovascular healthcare, and the person's awareness of disease and early onset of treatment are essential for effective management. ${ }^{11}$ Studies on dyslipidemia awareness, treatment and control and their determinants at national level in China are limited. Therefore, we aimed to estimate the awareness, treatment and control of dyslipidemia and their determinants among Chinese middle-aged and older adults using a nationally representative sample.

\section{Methods}

\section{STUDY POPULATION}

This cross-sectional study retrieved data from the 2015 CNSSPP. It was a national program instituted by the Chinese government in 2011 to support stroke prevention and control. Details have been described previously. ${ }^{12,13}$ Briefly, it involved Chinese adults aged $\geq 40$ years. It used a multistage probability-sampling strategy with random samples drawn from local areas, counties, rural and urban communities. ${ }^{13}$ Respondents were recruited from 200 project areas according to local population sizes and counties. Further, an urban and a rural community were chosen from each project area as primary sampling unit based on their geographical locations in the 30 municipalities and provinces across China.

\section{DATA COLLECTION}

Personnel were trained to take anthropometric measurements, record blood pressure (BP) and manage matters relating to the questionnaire. The questionnaire collected details of sociodemographic, medical and lifestyle information. Data examined included: age (in four categories, 40-49, 50-59, $60-69$, and $\geq 70$ years old); ${ }^{14}$ an urban area was defined using the 12-component urbanization index; ${ }^{15}$ level of education (primary school and below, junior/ middle school, senior/middle school, and college/higher); ${ }^{16}$ sex (male, female); ethnicity (Han, other ethnicities; ${ }^{17}$ marital status (married, widowed/divorced/unmarried). In addition were: health insurance coverage, classified into 'yes' and 'no' (yes refers to respondents on any health insurance program at the time of the interview). ${ }^{16}$ Socio-economic regions (high, middle, low-income) based on the per-capita disposable household's income thresholds in 2014. ${ }^{17}$ Current smoking (if a participant reported smoking at least one cigarette per day). Current drinking (if a participant reported drinking alcoholic beverages $\geq 1$ per week for more than half a year). ${ }^{17}$ Physical activity (if a participant reported an activity of $\geq 3$ times per week for at least 30 minutes each episode, or engaged in heavy physical work). ${ }^{16}$ History of stroke or CHD (a participant self-report of physician's diagnosis of CHD or stroke and/or currently receiving medications). ${ }^{17}$ Family history of dyslipidemia was on the basis of whether the respondent's family (parents and siblings) had been diagnosed by a physician for dyslipidemia. ${ }^{18}$. These data were obtained from self-reports of participants. Two geographical groupings were used to zone participants: a) the 
north and south zones (according to the Huai River-Qin Mountains Line), and b) the stroke and non-stroke belt zones. The stroke belt was defined based stroke incidence in China i.e. any region containing provinces that met the criteria for a region of high stroke incidence. ${ }^{17}$

Body weight, to the nearest $0.1 \mathrm{~kg}$, and height was measured, to the nearest $0.1 \mathrm{~cm}$. The body mass index (BMI) was calculated as the weight in kilograms divided by the height in meters squared $(\mathrm{kg} / \mathrm{m} 2)$ based on China's Ministry of Health criteria. ${ }^{19} \mathrm{BMl}$ categories (underweight if $\mathrm{BMI}<18.5 \mathrm{~kg} / \mathrm{m}^{2}$, normal weight $18.5-24.0 \mathrm{~kg} / \mathrm{m}^{2}$, overweight 24.0 to $<28 \mathrm{~kg} / \mathrm{m}^{2}$, and obesity $\geq 28 \mathrm{~kg} / \mathrm{m}^{2}$ ). Central obesity, was measured and defined as $\geq 0.90$ and $\geq 0.85$ for men and women, respectively. ${ }^{17}$

Blood pressure was measured three times and the average of the three readings was used to define hypertension as systolic BP $\geq 140 \mathrm{mmHg}$ or diastolic BP $\geq 90 \mathrm{mmHg}$, or self-report of physician diagnosis and currently receiving antihypertensive medications. ${ }^{20}$ Fasting glucose was used to define diabetes if $\geq 126 \mathrm{mg} / \mathrm{dL}(7.0 \mathrm{mmol} / \mathrm{L})$ or selfreport of physician diagnosis and currently receiving ant-diabetic medication.

A standardized protocol was used to collect blood samples from participants at all research centers. All laboratories received instructions on the protocols to be followed including labelling of kits for blood collection for each adult. Participants' venous blood samples were collected after 8-hour overnight fasting at tests centers with a lipid and a glucose tube, stored in cold boxes and transported to certified examination centers.

\section{DEFINITIONS OF LIPID VARIABLES}

Blood samples were investigated for TC, HDL-C, TG, and LDL-C according to standardized protocols. Lipid disorders were classified based on the latest Chinese guidelines for the prevention and treatment of dyslipidemia in adults. ${ }^{21}$ Dyslipidemia was defined as having either or combination of total cholesterol (TC) $\geq 240 \mathrm{mg} / \mathrm{dL}(6.22 \mathrm{mmol} / \mathrm{L})$ or LDLC $\geq 160 \mathrm{mg} / \mathrm{dL}(4.14 \mathrm{mmol} / \mathrm{L})$ or $\mathrm{HDL}-\mathrm{C}<40 \mathrm{mg} / \mathrm{dL}(1.04 \mathrm{mmol} / \mathrm{L})$, or triglyceride (TG) $\geq 200 \mathrm{mg} / \mathrm{dL}$ $(2.26 \mathrm{mmol} / \mathrm{L})$ or non-HDL-C $\geq 190 \mathrm{mg} / \mathrm{dl}(4.9 \mathrm{mmol} / \mathrm{L})$ or a current use of lipid modifying medications. ${ }^{21}$ Awareness of dyslipidemia was defined as a self-reported physician diagnosis of dyslipidemia or self-reported use of lipidlowering medication within the population defined as having dyslipidemia. In addition, treatment of dyslipidemia was defined as using prescribed lipid-lowering medications to treat dyslipidemia among participants with dyslipidemia, and control of dyslipidemia was defined as having dyslipidemia and being treated with medications if the individual has TC $<240 \mathrm{mg} / \mathrm{dL}, \mathrm{LDL}-\mathrm{C}<160 \mathrm{mg} / \mathrm{dL}, \mathrm{HDL}-\mathrm{C} \geq 40 \mathrm{mg} / \mathrm{dL}$ and triglycerides $<200 \mathrm{mg} / \mathrm{dL}$. $7,9,19$

\section{DATA ANALYSIS}

The International Business Machine Statistical Package for Social Sciences (IBM SPSS) version 19.0 (SPSS Inc, Chicago, III) software was used for all analyses. Continuous variables were presented as mean \pm SD (normal distribution) and as median(IQR) (skewed distribution). Categorical variables were reported as numbers (percentages), and by proportions and 95\% confidence intervals (Cls). The standardized awareness, treatment and control rates were calculated according to the age and sex-specific structure of the 2010 Chinese national population census. We explored the associations between determinants (categorical) and the outcome variables of interest (awareness, treatment, and control), using univariate and multivariable logistic models. Data were presented as unadjusted (OR) and adjusted odds ratios (aOR) and 95\% confidence intervals (Cl). All statistical tests were two-tailed and pvalues $\leq 0.05$ considered statistically significant. Processing of graphs and tables was done using MS excel 2013.

\section{Results}




\section{STUDY PARTICIPANTS}

About 180,000 participants were further randomly selected from all provinces purposely to assess them for stroke risk factors such as dyslipidemia. ${ }^{13}$ Of these, 135,403 weighted participants were finally used for the analysis after excluding people with missing and incomplete data related to BMI and lipid variables. Table 1 summarizes the distribution of participant's characteristics. Overall, $51.1 \%$ were women. The mean age (SD) was $56.56 \pm 9.98$ years. More than a third of respondents were aged 50-59 years (42.3\%). Approximately, sixty-two percent (62.1\%) of the subjects had primary or no education, and over $50 \%$ lived in rural areas (53.6\%). Most respondents (94.3\%), had a family history of dyslipidemia. Slightly over $40 \%$ of them were overweight, about $33 \%$ were current smokers, $19 \%$ drank alcohol, and $62 \%$ were physically active. About $62.4 \%$ of participants had central obesity. 
Table 1

Overall, dyslipidemic and non-dyslipidemic and characteristics among adults, China 2014

\begin{tabular}{|c|c|c|c|c|}
\hline Variables & Total & Dyslipidemic & Non-dyslipidemic & $\begin{array}{l}\mathrm{P} \\
\text { values }\end{array}$ \\
\hline Total & 135,403 (100\%) & $57760(42.7 \%)$ & $77642(57.3 \%)$ & \\
\hline Age, & $56.56 \pm 9.98$ & $56.48 \pm 9.73$ & $56.62 \pm 10.16$ & 0.12 \\
\hline \multicolumn{5}{|l|}{ Median (IQR) } \\
\hline $40-49$ & $35617(26.3)$ & $14821(25.7)$ & $20796(26.8)$ & $<0.001$ \\
\hline $50-59$ & $57300(42.3)$ & $25037(43.3)$ & $32263(41.6)$ & \\
\hline $60-69$ & $26154(19.3)$ & $11437(19.8)$ & $14717(19.0)$ & \\
\hline 70 and above & $16331(12.1)$ & 6465 (11.2) & $9866(12.7)$ & \\
\hline \multicolumn{5}{|l|}{ Sex } \\
\hline Men & $66259(48.9)$ & $27816(48.2)$ & $38443(49.5)$ & $<0.001$ \\
\hline Women & $69144(51.1)$ & $29945(51.8)$ & $39199(50.5)$ & \\
\hline \multicolumn{5}{|l|}{ Residence } \\
\hline Rural & $72556(53.6)$ & $31292(54.2)$ & $41264(53.1)$ & $<0.001$ \\
\hline Urban & $62847(46.4)$ & $26469(45.8)$ & $36378(46.9)$ & \\
\hline \multicolumn{5}{|l|}{ Nationality } \\
\hline Han & $130934(96.7)$ & $55557(96.2)$ & 75377 (97.1) & $<0.001$ \\
\hline Others & 4465 (3.3) & $22.3(3.8)$ & $2262(2.9)$ & \\
\hline \multicolumn{5}{|l|}{ Marital Status } \\
\hline Married & $80154(59.2)$ & $32362(56.0)$ & $47792(61.6)$ & $<0.001$ \\
\hline Divorced/Widowed/Others & $55248(40.8)$ & $25398(44.0)$ & $29850(38.4)$ & \\
\hline \multicolumn{5}{|l|}{ Level of Education } \\
\hline Primary and Below & $84118(62.1)$ & $37459(64.9)$ & $46659(60.1)$ & $<0.001$ \\
\hline Junior/Middle School & $32233(23.8)$ & $12264(21.2)$ & $19969(25.7)$ & \\
\hline Senior High School & $13104(9.7)$ & $5598(9.7)$ & 7506 (9.7) & \\
\hline College and above & $5948(4.4)$ & $2440(4.2)$ & $3508(4.5)$ & \\
\hline \multicolumn{5}{|l|}{ Health Insurance } \\
\hline Yes & $104254(77.0)$ & $49092(85.0)$ & $55162(71.0)$ & $<0.001$ \\
\hline No & $31149(23.0)$ & 8669 (15.0) & $22480(29.0)$ & \\
\hline \multicolumn{5}{|l|}{ Socio- economic region } \\
\hline Low-income & 32084 (23.7) & $15029(26.0)$ & $17055(22.0)$ & $<0.001$ \\
\hline
\end{tabular}




\begin{tabular}{|c|c|c|c|c|}
\hline Variables & Total & Dyslipidemic & Non-dyslipidemic & $\begin{array}{l}P \\
\text { values }\end{array}$ \\
\hline Middle- income & $40212(29.7)$ & $18395(31.8)$ & $21817(28.1)$ & \\
\hline High-income & 63108 (46.6) & $24337(42.1)$ & 38771 (49.9) & \\
\hline \multicolumn{5}{|l|}{ Geographical Regions } \\
\hline 1 North & $75174(55.5)$ & $29172(50.5)$ & $46002(59.2)$ & $<0.001$ \\
\hline South & $60229(44.5)$ & $28589(49.5)$ & $31640(40.8)$ & \\
\hline \multicolumn{5}{|l|}{ Stroke Belt Zone } \\
\hline Yes & $23262(17.2)$ & $9449(16.4)$ & $13813(17.8)$ & $<0.001$ \\
\hline No & $112142(82.8)$ & $48312(83.6)$ & $63830(82.2)$ & \\
\hline Weight & $\begin{array}{l}65.00(58.00- \\
71.50)\end{array}$ & $\begin{array}{l}\text { 65.00 (58.00- } \\
72.00)\end{array}$ & $\begin{array}{l}55.00(49.00- \\
63.00)\end{array}$ & $<0.001$ \\
\hline Height & $162.07 \pm 8.40$ & $161.85 \pm 8.56$ & $162.24 \pm 8.27$ & $<0.001$ \\
\hline \multicolumn{5}{|l|}{ BMla } \\
\hline Median (IQR) & $\begin{array}{l}25.2(23.02- \\
27.47)\end{array}$ & $25.7(23.46-27.89)$ & $\begin{array}{l}25.05(22.66- \\
27.09)\end{array}$ & \\
\hline Underweight $(<18.5 \mathrm{~kg} / \mathrm{m} 2$ & $1835(1.4)$ & $568(1.0)$ & $1267(1.6)$ & $<0.001$ \\
\hline Normal $(18.5-<24.0)$ & $46598(34.4)$ & $16968(29.4)$ & $29630(38.2)$ & \\
\hline Overweight $(24.0<28)$ & $59314(43.8)$ & $26232(45.4)$ & $33082(42.6)$ & \\
\hline Obesity ( $\geq 28.0$ ) & $27655(20.4$ & $13992(24.2)$ & $13663(17.6)$ & \\
\hline \multicolumn{5}{|l|}{ Waist Circumference $^{\dagger}$} \\
\hline Median (IQR) & $\begin{array}{l}86.00(80.00- \\
93.00)\end{array}$ & $88.0(80.00-95.00)$ & $\begin{array}{l}85.00(79.00- \\
92.00)\end{array}$ & \\
\hline Normal < 90/85 cm (M/W) & $56214(42.0)$ & $21034(36.7)$ & $35180(45.9)$ & $<0.001$ \\
\hline Central obese $\geq 90 / 85 \mathrm{~cm}(\mathrm{M} / \mathrm{W})$ & $77700(58.0)$ & $36230(63.3)$ & $41470(54.1)$ & $<0.001$ \\
\hline Family History of Dyslipidemia & $127624(94.3)$ & $53695(93.0)$ & $3714(4.8)$ & $<0.001$ \\
\hline History of CHD & $123279(91.0)$ & $51455(89.1)$ & $71824(92.5)$ & $<0.001$ \\
\hline History of Stroke & $107268(79.2)$ & $44726(77.4)$ & $62542(80.6)$ & $<0.001$ \\
\hline $\begin{array}{l}\text { Fasting Blood } \\
\text { Glucose(FBG)mmol/I }\end{array}$ & $5.94 \pm 1.91$ & $5.95 \pm 1.95$ & $5.94 \pm 1.88$ & $<0.001$ \\
\hline Systolic Blood Pressure (SBP) & $94.22 \pm 63.66$ & $98.34 \pm 63.29$ & $91.16 \pm 63.77$ & $<0.001$ \\
\hline Diastolic Blood Pressure (DBP) & $57.80 \pm 39.00$ & $59.84 \pm 38.36$ & $56.28 \pm 39.39$ & $<0.001$ \\
\hline Diabetes Mellitus & $41036(30.3)$ & $19059(33.0)$ & $21977(28.3)$ & $<0.001$ \\
\hline Hypertension & $84462(62.4)$ & 37634 (65.2) & $46828(60.3)$ & $<0.001$ \\
\hline
\end{tabular}




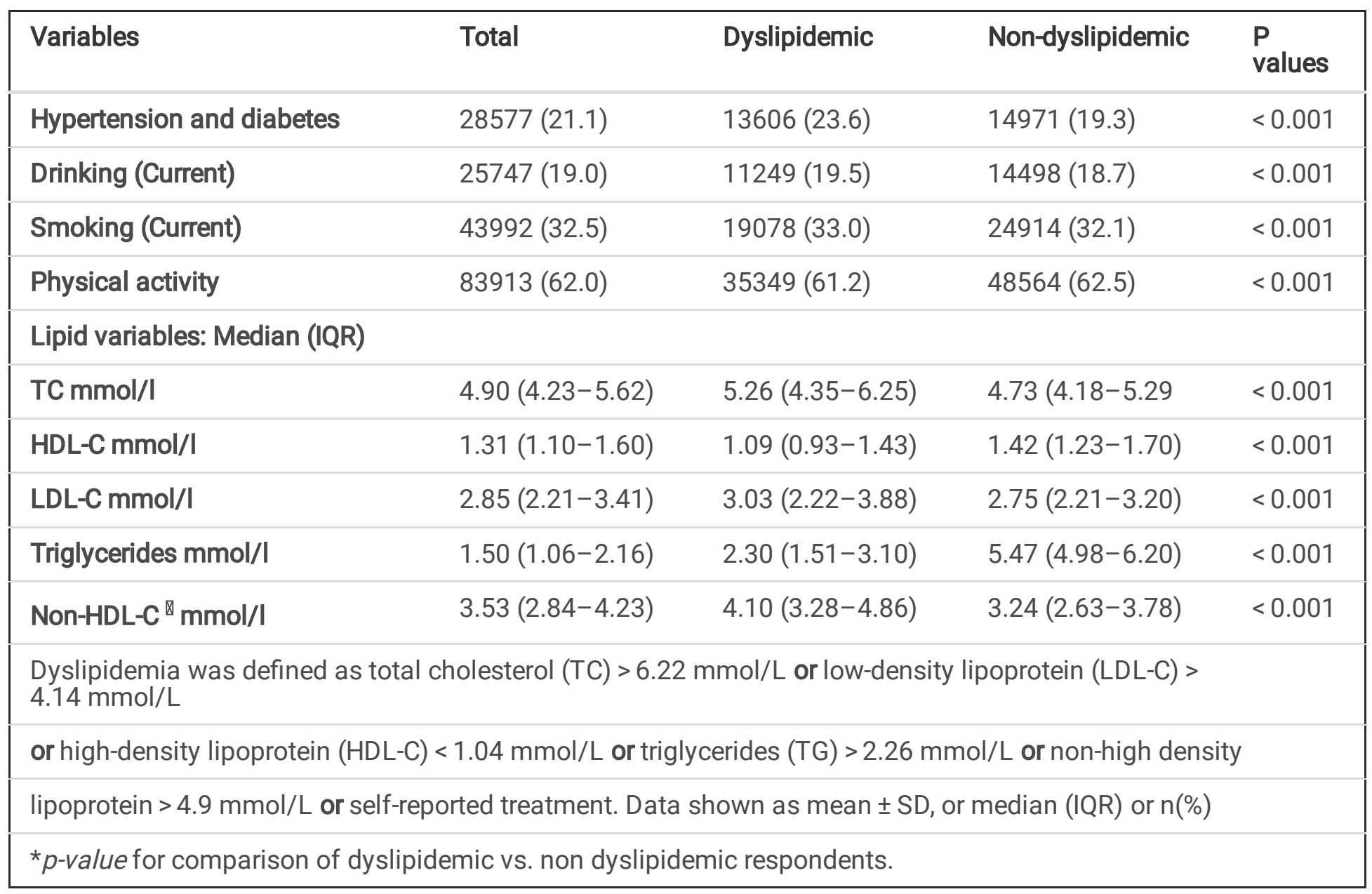

AWARENESS, TREATMENT AND CONTROL OF DYSLIPIDEMIA

A previous study by Opoku et al. with a sample of 136,945 Chinese adults' aged $\geq 40$ years showed that the prevalence of dyslipidemia was $43 \%$. In this study, as shown in Table 2, a total of 36,958 (64.0\%) persons were aware of the condition, of whom 6993 (18.9\%) received treatment, and of whom 505 (7.3\%) had controlled lipids.

Considering the age groups, the highest proportion of awareness (64.3\%) was seen among the $\geq 70$ year olds, adults aged $60-69$ years had the highest proportion for treatment $(19.2 \%)$, whilst lipids control was highest $(9.8 \%)$ among 40-49 years old individuals. Women versus men, respectively, had higher proportions of awareness, treatment, and control $(69.2 \%$ vs. $58.3 \%, 23.6 \%$ vs. $12.9 \%$, and $8.3 \%$ vs. $4.7 \%)$. 
Table 2

Awareness, treatment and control of dyslipidemia and their determinants among Adults, China 2014

\begin{tabular}{|c|c|c|c|c|}
\hline Variable & & Awareness & Treatment & Control \\
\hline Total & & $36958(64 \%)$ & $6993(18.9 \%)$ & $504(7.2 \%)$ \\
\hline \multirow[t]{5}{*}{ Age group (years) } & $40-49$ & $64.2(63.4-64.9)$ & $19.1(12.3-27.6)$ & $9.8(5.1-16.8)$ \\
\hline & $50-59$ & $63.7(63.1-64.3)$ & $18.8(12.1-27.3)$ & $6.8(3.1-13.0)$ \\
\hline & $60-69$ & $64.2(63.3-65.1)$ & $19.2(12.4-27.7)$ & $5.2(2.1-10.9)$ \\
\hline & 70 and above & $64.3(63.1-65.4)$ & $18.3(11.7-26.7)$ & $6.6(2.9-12.7)$ \\
\hline & & $P=0.718$ & $P=0.632$ & $P<0.001$ \\
\hline \multirow[t]{3}{*}{ Sex } & Men & $58.3(57.8-58.9)$ & $12.9(7.4-20.5)$ & $4.7(1.8-10.2)$ \\
\hline & Women & $69.2(68.7-69.7)$ & $23.6(16.1-32.6)$ & $8.3(4.1-14.9)$ \\
\hline & & $P<0.001$ & $P<0.001$ & $P<0.001$ \\
\hline \multirow[t]{3}{*}{ Residence } & Rural & $61.0(60.5-61.6)$ & $18.6(11.9-27.1)$ & $8.2(4.0-14.8)$ \\
\hline & Urban & $67.5(66.9-68.1)$ & $19.3(12.5-27.8)$ & $6.2(2.7-12.2)$ \\
\hline & & $P<0.001$ & $P=0.081$ & $P<0.001$ \\
\hline \multirow[t]{3}{*}{ Ethnicity } & Han & )$^{67.5(66.9-68.1}$ & $19.0(12.3-27.5)$ & $7.3(3.4-13.6)$ \\
\hline & Others & $\begin{array}{l}61.0(60.5- \\
61.6)\end{array}$ & $16.8(10.5-25.0)$ & $4.3(1.5-9.6)$ \\
\hline & & $P<0.001$ & $P=0.040$ & $P=0.077$ \\
\hline \multirow[t]{3}{*}{ Marital Status } & Married & $63.8(63.3-64.3)$ & $19.5(12.7-28.1)$ & $7.0(3.2-13.3)$ \\
\hline & Divorced/Widowed/Others & $64.2(63.6-68.2)$ & $18.2(11.6-26.6)$ & $7.5(3.5-13.9)$ \\
\hline & & $P=0.367$ & $P<0.001$ & $P=0.0 .371$ \\
\hline \multirow[t]{5}{*}{ Level of Education } & Primary and Below & $62.6(62.1-63.1)$ & $18.4(11.8-26.8)$ & $7.3(3.4-13.6)$ \\
\hline & Junior/Middle School & $65.6(64.8-66.4)$ & $20.1(13.2-28.7)$ & $7.2(3.3-13.5)$ \\
\hline & Senior High School & $67.0(65.8-69.8)$ & $19.3(12.5-27.8)$ & $6.7(3.0-12.9)$ \\
\hline & College and above & $69.5(67.7-71.3)$ & $19.6(12.7-28.2)$ & $7.2(3.3-13.5)$ \\
\hline & & $P<0.001$ & $P=0.001$ & $P=0.961$ \\
\hline \multirow[t]{3}{*}{ Health Insurance } & Yes & $63.4(62.9-63.8)$ & $19.1(12.3-27.6)$ & $6.2(2.7-12.2)$ \\
\hline & No & $67.5(66.5-68.5)$ & $17.8(11.3-26.2)$ & $12.8(7.3-20.4)$ \\
\hline & & $P<0.001$ & $P=0.020$ & $P<0.001$ \\
\hline \multirow{2}{*}{$\begin{array}{l}\text { Socio- economic } \\
\text { region }\end{array}$} & Low- income & $63.3(62.5-64.0)$ & $17.3(10.9-25.6)$ & $5.3(2.1-11.0)$ \\
\hline & Middle- income & $66.1(65.4-66.8)$ & $17.4(11.0-25.7)$ & $6.8(3.1-13.0)$ \\
\hline
\end{tabular}




\begin{tabular}{|c|c|c|c|c|}
\hline Variable & & Awareness & Treatment & Control \\
\hline & High-income & $62.8(62.2-63.4)$ & $21.1(14.0-29.8)$ & $8.5(4.2-15.2)$ \\
\hline \multirow[t]{3}{*}{ Geographical Regions } & North & $63.1(53.4-72.1)$ & $19.5(12.7-28.1)$ & $8.7(4.3-15.4)$ \\
\hline & South & $64.8(55.1-73.6)$ & $18.4(11.8-26.8)$ & $5.6(2.3-11.4)$ \\
\hline & & $P<0.001$ & $P=0.009$ & $P<0.001$ \\
\hline \multirow[t]{3}{*}{ Stroke Belt } & Yes & $64.0(54.3-72.9)$ & $18.0(11.4-26.4)$ & $5.0(1.9-10.6)$ \\
\hline & No & $64.8(55.1-73.6)$ & $19.1(12.3-27.6)$ & $7.6(3.6-14.0)$ \\
\hline & & $P<0.001$ & $P=0.069$ & $P=0.003$ \\
\hline \multirow[t]{5}{*}{ BMI } & $\begin{array}{l}\text { Underweight (< } \\
18.5 \mathrm{~kg} / \mathrm{m} 2\end{array}$ & $64.6(63.9-65.3)$ & $18.3(11.7-26.7)$ & $8.5(4.2-15.2)$ \\
\hline & Normal $(18.5-24.0)$ & $62.1(58.1-66.1)$ & $14.4(8.6-22.3)$ & $7.8(3.7-14.3)$ \\
\hline & Overweight $(24.0<28)$ & $63.6(63.0-64.2)$ & $19.2(12.4-27.7)$ & $6.9(3.1-13.1)$ \\
\hline & Obesity ( $\geq 28.0$ ) & $64.0(63.2-64.8)$ & $19.4(12.6-28.0)$ & $6.4(2.8-12.5)$ \\
\hline & & $P=0.179$ & $P=0.032$ & $P=0.075$ \\
\hline \multirow[t]{2}{*}{ Waist Circumference } & Normal < 90/85 cm & $62.3(52.6-71.3)$ & $17.7(11.2-26.0)$ & $7.3(3.4-13.6)$ \\
\hline & Central obese $\geq 90 / 85 \mathrm{~cm}$ & $64.9(55.6-73.7)$ & $19.7(12.8-28.3)$ & $7.1(3.3-13.4)$ \\
\hline Family History & Yes & $66.2(56.6-74.9)$ & $18.9(12.2-27.4)$ & $7.3(3.4-13.6)$ \\
\hline \multirow[t]{2}{*}{ of Dyslipidemia } & No & $34.6(25.8-44.3)$ & $\begin{array}{l}19.7(12.8- \\
28.3)\end{array}$ & $4.3(1.5-9.6)$ \\
\hline & & $P<0.001$ & $P=0.438$ & $P=0.061$ \\
\hline \multirow[t]{3}{*}{ History of Stroke } & Yes & $66.2(56.6-74.9)$ & $20.0(13.1-28.6)$ & $7.5(3.5-13.9)$ \\
\hline & No & $56.4(46.6-65.8)$ & $14.6(8.7-22.5)$ & $5.6(2.3-11.4)$ \\
\hline & & $P<0.001$ & $P<0.001$ & $P=0.028$ \\
\hline \multirow[t]{3}{*}{ History of CHD } & Yes & $65.8(56.2-74.5)$ & $19.3(12.5-27.8)$ & $7.2(3.3-13.5)$ \\
\hline & No & $49.5(39.8-59.2)$ & $15.0(9.0-23.0)$ & $7.7(3.7-14.1)$ \\
\hline & & $P<0.001$ & $P<0.001$ & $P=0674$ \\
\hline \multirow[t]{3}{*}{ Diabetes Mellitus } & Yes & $64.4(54.7-73.3)$ & $18.0(11.4-26.4)$ & $7.6(3.6-14.0)$ \\
\hline & No & $63.8(54.1-72.7)$ & $19.4(12.6-28.0)$ & $7.0(3.2-13.3)$ \\
\hline & & $P=0.177$ & $P<0.001$ & $P=0.378$ \\
\hline \multirow[t]{3}{*}{ Hypertension } & Yes & $\begin{array}{l}64.1(54.4-73 . \\
0)\end{array}$ & $64.1(54.4-73.0)$ & $7.0(3.2-13.3)$ \\
\hline & No & $63.8(54.1-72.7)$ & $63.8(54.1-72.7)$ & $7.7(3.7-14.1)$ \\
\hline & & $P=0.452$ & $P=0.452$ & $P=0.281$ \\
\hline
\end{tabular}




\begin{tabular}{|lllll|}
\hline Variable & & Awareness & Treatment & Control \\
\hline Drinking (Current) & Yes & $68.6(59.1-77.1)$ & $68.6(59.1-77.1)$ & $6.3(2.7-12.3)$ \\
& No & $62.9(53.2-71.9)$ & $62.9(53.2-71.9)$ & $7.6(3.6-14.0)$ \\
\hline Smoking (Current) & Yes & $P<0.001$ & $P<0.001$ & $P=0.058$ \\
& No & $68.6(59.1-77.1)$ & $68.6(59.1-77.1)$ & $7.6(3.6-14.0)$ \\
\hline & & $61.7(51.9-70.8)$ & $61.7(51.9-70.8)$ & $6.9(3.1-13.1)$ \\
\hline Physical activity & Yes & $P<0.001$ & $P<0.001$ & $P=0.279$ \\
& No & $64.1(54.4-73.0)$ & $64.1(54.4-73.0)$ & $7.6(3.6-14.0)$ \\
\hline & & $63.8(54.1-72.7)$ & $63.8(54.1-72.7)$ & $6.6(2.9-12.7)$ \\
\hline
\end{tabular}

Figure 1 shows that the proportion of dyslipidemia awareness among women in rural and urban settings was similar ( $69 \%$ vs. $69.5 \%)$, but dissimilar in urban $(65.3 \%)$ than in rural men $(52.4 \%),(p<0.001)$. Treatment was higher in men living in rural compared to urban areas (15.4\% vs. $10.6 \%)$. Unlike men, urban women compared with their rural counterparts had greater treatment ( $26.9 \%$ vs. $20.8 \%)$. The proportion of control was seen more in women than in men of both residences.

As shown in Fig. 2 the proportions of awareness were more among urban compared with rural residents across all the three socioeconomic regions: low-income ( $68.8 \%$ vs. $60.4 \%)$, middle-income ( $69.4 \%$ vs. $63.9 \%)$, and high-income ( $66 \%$ vs. $58.6 \%)$, respectively $(p<0.001)$. Rural versus urban adults showed higher treatment results in two socioeconomic regions namely: low - income ( $18.3 \%$ vs. $15.8 \%)$, and high-income ( $20.8 \%$ vs. $19.3 \%)$. The proportion of dyslipidemia control in rural areas was higher in all socioeconomic regions: low -income (6.3\% vs. $3.4 \%)$, middleincome ( $9.4 \%$ vs. $3.3 \%)$, and high-income $(8.7 \%$ vs. $8.3 \%)$, (all p $<0.001)$.

Women recorded significantly higher dyslipidemia proportions for awareness, treatment and control in all socioeconomic regions $(p<0.001)$ Fig. 3.

\section{DETERMINANTS OF AWARENESS, TREATMENT, AND CONTROL OF DYSLIPIDEMIA.}

Table 3 shows the CORs and adjusted ORs of the determinants for dyslipidemia awareness. In the bivariate analysis, we found positive association between dyslipidemia awareness and the following determinants: education levels, current smoking, living in a middle-income region, and current drinking. However, having a health insurance and living in a northern zone were related to lower odds for awareness. In the adjusted model, being overweight (AOR: $0.92,95 \%$ Cl: $0.89-0.97$ ) and living in a stroke belt zone (AOR: $0.80,95 \% \mathrm{Cl}: 0.76-0.84$ ) showed lower likelihoods of dyslipidemia awareness. 
Table 3

Univariate and multivariate analyses of factors affecting awareness of dyslipidemia among adults. * Adjusted for all variables cited in the table

\begin{tabular}{|c|c|c|c|c|c|c|c|c|}
\hline \multirow[t]{2}{*}{ Variable } & & & \multicolumn{2}{|c|}{ Awareness } & \multicolumn{2}{|c|}{ Treatment } & \multicolumn{2}{|l|}{ Control } \\
\hline & & & $\begin{array}{l}\text { COR } \\
(95 \% \mathrm{Cl})\end{array}$ & $\begin{array}{l}* \mathrm{aOR} \\
(95 \% \mathrm{Cl})\end{array}$ & $\begin{array}{l}\text { COR } \\
(95 \% \mathrm{Cl})\end{array}$ & $\begin{array}{l}* \mathrm{aOR} \\
(95 \% \mathrm{Cl})\end{array}$ & $\begin{array}{l}\text { COR } \\
(95 \% \mathrm{Cl})\end{array}$ & $\begin{array}{l}* \mathrm{aOR} \\
(95 \% \mathrm{Cl})\end{array}$ \\
\hline & & $50-59$ & $\begin{array}{l}0.98 \\
(0.94- \\
1.02)\end{array}$ & & $\begin{array}{l}0.99 \\
(0.94- \\
1.05)\end{array}$ & & $\begin{array}{l}0.67 \\
(0.54- \\
0.82)\end{array}$ & $\begin{array}{l}0.69 \\
(0.55- \\
0.85)\end{array}$ \\
\hline \multirow[t]{2}{*}{ Age } & $\begin{array}{l}\text { (Ref: } 40- \\
49)\end{array}$ & $60-69$ & $\begin{array}{l}1.00 \\
(0.95- \\
1.05)\end{array}$ & & $\begin{array}{l}1.02 \\
(0.96- \\
1.09)\end{array}$ & & $\begin{array}{l}0.51 \\
(0.38- \\
0.67)\end{array}$ & $\begin{array}{l}0.53 \\
(0.40- \\
0.70)\end{array}$ \\
\hline & & 70 and above & $\begin{array}{l}1.00 \\
(0.94- \\
1.07)\end{array}$ & & $\begin{array}{l}0.98 \\
(0.91- \\
1.06)\end{array}$ & & $\begin{array}{l}0.64 \\
(0.47- \\
0.89)\end{array}$ & $\begin{array}{l}0.68 \\
(0.49- \\
0.95)\end{array}$ \\
\hline Sex & \multicolumn{2}{|l|}{ (Ref: men) } & $\begin{array}{l}1.61 \\
(1.55- \\
1.66)\end{array}$ & $\begin{array}{l}1.53 \\
(1.46- \\
1.59)\end{array}$ & $\begin{array}{l}1.89 \\
(1.81- \\
1.97)\end{array}$ & $\begin{array}{l}1.78 \\
(1.69- \\
1.88)\end{array}$ & $\begin{array}{l}1.83 \\
(1.46- \\
2.29)\end{array}$ & $\begin{array}{l}1.64 \\
(1.30- \\
2.07)\end{array}$ \\
\hline Residence & \multicolumn{2}{|l|}{ (Ref: rural) } & $\begin{array}{l}1.33 \\
(1.28- \\
1.37)\end{array}$ & $\begin{array}{l}1.40 \\
(1.35- \\
1.45)\end{array}$ & $\begin{array}{l}1.00 \\
(0.96- \\
1.05)\end{array}$ & & $\begin{array}{l}0.74 \\
(0.62- \\
0.89)\end{array}$ & $\begin{array}{l}0.68 \\
(0.56- \\
0.83)\end{array}$ \\
\hline Nationality & $\begin{array}{l}\text { (Ref: } \\
\text { others) }\end{array}$ & & $\begin{array}{l}1.07 \\
(0.95- \\
1.13)\end{array}$ & & $\begin{array}{l}1.07 \\
(0.96- \\
1.20)\end{array}$ & & $\begin{array}{l}1.74 \\
(0.92- \\
3.28)\end{array}$ & \\
\hline \multirow[t]{2}{*}{ Marital Status } & \multicolumn{2}{|c|}{$\begin{array}{l}\text { (Ref: } \\
\text { Divorced/Widowed/Others) }\end{array}$} & $\begin{array}{l}0.98 \\
(0.95- \\
1.02)\end{array}$ & & $\begin{array}{l}1.06 \\
(1.02- \\
1.11)\end{array}$ & $\begin{array}{l}1.00 \\
(0.96- \\
1.08)\end{array}$ & $\begin{array}{l}0.92 \\
(0.77- \\
1.10)\end{array}$ & \\
\hline & & $\begin{array}{l}\text { Junior/Middle } \\
\text { School }\end{array}$ & $\begin{array}{l}1.14 \\
(1.09- \\
1.19)\end{array}$ & $\begin{array}{l}1.05 \\
(1.00- \\
1.10)\end{array}$ & $\begin{array}{l}1.10 \\
(1.04- \\
1.16)\end{array}$ & $\begin{array}{l}1.02 \\
(0.96- \\
1.08)\end{array}$ & $\begin{array}{l}0.98 \\
(0.79- \\
1.22)\end{array}$ & \\
\hline \multirow[t]{2}{*}{$\begin{array}{l}\text { Level of } \\
\text { Education }\end{array}$} & $\begin{array}{l}\text { (Ref: } \\
\text { Primary } \\
\text { and } \\
\text { Below) }\end{array}$ & $\begin{array}{l}\text { Senior High } \\
\text { School }\end{array}$ & $\begin{array}{l}1.21 \\
(1.14- \\
1.29)\end{array}$ & $\begin{array}{l}1.03 \\
(0.96- \\
1.09)\end{array}$ & $\begin{array}{l}1.07 \\
(1.00- \\
1.15)\end{array}$ & $\begin{array}{l}1.00 \\
(0.92- \\
1.08)\end{array}$ & $\begin{array}{l}0.92 \\
(0.67- \\
1.25)\end{array}$ & \\
\hline & & $\begin{array}{l}\text { College and } \\
\text { above }\end{array}$ & $\begin{array}{l}1.36 \\
(1.25- \\
1.49)\end{array}$ & $\begin{array}{l}1.05 \\
(0.95- \\
1.16)\end{array}$ & $\begin{array}{l}1.15 \\
(1.03- \\
1.27)\end{array}$ & $\begin{array}{l}1.02 \\
(0.91- \\
1.13)\end{array}$ & $\begin{array}{l}0.99 \\
(0.64- \\
1.52)\end{array}$ & \\
\hline $\begin{array}{l}\text { Health } \\
\text { Insurance }\end{array}$ & \multicolumn{2}{|l|}{ (Ref: no) } & $\begin{array}{l}0.83 \\
(0.79- \\
0.88)\end{array}$ & $\begin{array}{l}1.09 \\
(1.03- \\
1.14)\end{array}$ & $\begin{array}{l}1.07 \\
(1.01- \\
1.13)\end{array}$ & $\begin{array}{l}1.12 \\
(1.05- \\
1.19)\end{array}$ & $\begin{array}{l}0.46 \\
(0.37- \\
0.56)\end{array}$ & $\begin{array}{l}0.50 \\
(0.41- \\
0.62)\end{array}$ \\
\hline \multirow[t]{2}{*}{$\begin{array}{l}\text { Socio- } \\
\text { economic } \\
\text { region }\end{array}$} & $\begin{array}{l}\text { (Ref: Low- } \\
\text { income) }\end{array}$ & $\begin{array}{l}\text { Middle- } \\
\text { income }\end{array}$ & $\begin{array}{l}1.13 \\
(1.08- \\
1.19)\end{array}$ & $\begin{array}{l}1.04 \\
(0.99- \\
1.09)\end{array}$ & $\begin{array}{l}0.98 \\
(0.92- \\
1.03)\end{array}$ & $\begin{array}{l}0.94 \\
(0.89- \\
1.00)\end{array}$ & $\begin{array}{l}1.31 \\
(0.99- \\
1.72)\end{array}$ & $\begin{array}{l}1.01 \\
(0.76- \\
1.36)\end{array}$ \\
\hline & & High-income & $\begin{array}{l}0.98 \\
(0.94- \\
1.02)\end{array}$ & $\begin{array}{l}0.88 \\
(0.84- \\
0.93)\end{array}$ & $\begin{array}{l}1.22 \\
(1.15- \\
1.28)\end{array}$ & $\begin{array}{l}1.24 \\
(1.17- \\
1.31)\end{array}$ & $\begin{array}{l}1.66 \\
(1.29- \\
2.12)\end{array}$ & $\begin{array}{l}1.25 \\
(0.95- \\
1.67)\end{array}$ \\
\hline $\begin{array}{l}\text { Geographical } \\
\text { Regions }\end{array}$ & \multicolumn{2}{|l|}{$\begin{array}{l}\text { (Ref: } \\
\text { South) }\end{array}$} & $\begin{array}{l}0.93 \\
(0.90- \\
0.96)\end{array}$ & $\begin{array}{l}1.03 \\
(0.98- \\
1.07)\end{array}$ & $\begin{array}{l}1.02 \\
(0.98- \\
1.06)\end{array}$ & & $\begin{array}{l}1.59 \\
(1.32- \\
1.92)\end{array}$ & $\begin{array}{l}1.74 \\
(1.40- \\
2.16)\end{array}$ \\
\hline
\end{tabular}




\begin{tabular}{|c|c|c|c|c|c|c|c|c|}
\hline \multicolumn{3}{|l|}{ Variable } & \multicolumn{2}{|c|}{ Awareness } & \multicolumn{2}{|c|}{ Treatment } & \multicolumn{2}{|l|}{ Control } \\
\hline Stroke Belt & $\begin{array}{l}\text { (Ref: Non- } \\
\text { stroke Belt }\end{array}$ & & $\begin{array}{l}0.80 \\
(0.77- \\
0.84)\end{array}$ & $\begin{array}{l}0.80 \\
(0.76- \\
0.84)\end{array}$ & $\begin{array}{l}0.91 \\
(0.86- \\
0.97)\end{array}$ & $\begin{array}{l}0.86 \\
(0.81- \\
0.91)\end{array}$ & $\begin{array}{l}0.64 \\
(0.47- \\
0.86)\end{array}$ & $\begin{array}{l}0.54 \\
(0.39- \\
0.74)\end{array}$ \\
\hline & & $\begin{array}{l}\text { Underweight } \\
(<18.5 \mathrm{~kg} / \mathrm{m} 2\end{array}$ & $\begin{array}{l}0.90 \\
(0.76- \\
1.07)\end{array}$ & $\begin{array}{l}0.97 \\
(0.8- \\
-1.16)\end{array}$ & $\begin{array}{l}0.84 \\
(0.67- \\
1.06)\end{array}$ & $\begin{array}{l}0.87 \\
(0.89- \\
1.00)\end{array}$ & $\begin{array}{l}0.94 \\
(0.34- \\
2.62)\end{array}$ & $\begin{array}{l}1.13 \\
(0.40- \\
3.22)\end{array}$ \\
\hline \multirow[t]{2}{*}{ BMI } & $\begin{array}{l}\text { (Ref: } \\
\text { Normal } \\
(18.5 \leq \\
24.0)\end{array}$ & $\begin{array}{l}\text { Overweight } \\
(24.0-<28)\end{array}$ & $\begin{array}{l}0.96 \\
(0.92- \\
0.99)\end{array}$ & $\begin{array}{l}0.92 \\
(0.89- \\
0.97)\end{array}$ & $\begin{array}{l}1.07 \\
(1.02- \\
1.13)\end{array}$ & $\begin{array}{l}1.04 \\
(0.98- \\
1.09)\end{array}$ & $\begin{array}{l}0.80 \\
(0.65- \\
0.99)\end{array}$ & $\begin{array}{l}0.78 \\
(0.63- \\
0.97)\end{array}$ \\
\hline & & $\begin{array}{l}\text { Obesity ( } \geq \\
28.0 \text { ) }\end{array}$ & $\begin{array}{l}0.98 \\
(0.93- \\
1.02)\end{array}$ & $\begin{array}{l}0.95 \\
(0.90- \\
1.00)\end{array}$ & $\begin{array}{l}1.09 \\
(1.03- \\
1.15)\end{array}$ & $\begin{array}{l}1.06 \\
(0.99- \\
1.13)\end{array}$ & $\begin{array}{l}0.74 \\
(0.58- \\
0.95)\end{array}$ & $\begin{array}{l}0.73 \\
(0.57- \\
0.94)\end{array}$ \\
\hline $\begin{array}{l}\text { Waist } \\
\text { Circumference }\end{array}$ & $\begin{array}{l}\text { (Ref: } \\
\text { Normal < } \\
90 / 85 \text { cm) }\end{array}$ & & $\begin{array}{l}1.12 \\
(1.08- \\
1.16)\end{array}$ & $\begin{array}{l}1.04 \\
(1.00- \\
1.09)\end{array}$ & $\begin{array}{l}1.15 \\
(1.10- \\
1.20)\end{array}$ & $\begin{array}{l}0.99 \\
(0.94- \\
1.04)\end{array}$ & $\begin{array}{l}0.96 \\
(0.80- \\
1.17)\end{array}$ & \\
\hline $\begin{array}{l}\text { History of } \\
\text { CHD }\end{array}$ & (Ref: no) & & $\begin{array}{l}1.96 \\
(1.86- \\
2.06)\end{array}$ & $\begin{array}{l}1.41 \\
(1.33- \\
1.49)\end{array}$ & $\begin{array}{l}1.24 \\
(1.15- \\
1.33)\end{array}$ & $\begin{array}{l}1.13 \\
(1.05- \\
1.21)\end{array}$ & $\begin{array}{l}0.92 \\
(0.65- \\
1.30)\end{array}$ & \\
\hline $\begin{array}{l}\text { Family History } \\
\text { of } \\
\text { Dyslipidemia }\end{array}$ & (Ref: no) & & $\begin{array}{l}3.71 \\
(3.47- \\
3.97)\end{array}$ & $\begin{array}{l}3.21 \\
(2.99- \\
3.45)\end{array}$ & $\begin{array}{l}0.94 \\
(0.87- \\
1.02)\end{array}$ & & $\begin{array}{l}1.67 \\
(0.94- \\
2.98)\end{array}$ & \\
\hline $\begin{array}{l}\text { History of } \\
\text { Stroke }\end{array}$ & (Ref: no) & & $\begin{array}{l}1.51 \\
(1.46- \\
1.58)\end{array}$ & $\begin{array}{l}1.20 \\
(1.15- \\
1.25)\end{array}$ & $\begin{array}{l}1.33 \\
(1.26- \\
1.40)\end{array}$ & $\begin{array}{l}1.23 \\
(1.17- \\
1.30)\end{array}$ & $\begin{array}{l}1.37 \\
(1.03- \\
1.80)\end{array}$ & $\begin{array}{l}1.23 \\
(0.93- \\
1.62)\end{array}$ \\
\hline $\begin{array}{l}\text { Diabetes } \\
\text { Mellitus }\end{array}$ & (Ref: no) & & $\begin{array}{l}1.03 \\
(0.99- \\
1.06)\end{array}$ & & $\begin{array}{l}0.93 \\
(0.89- \\
0.97)\end{array}$ & $\begin{array}{l}0.94 \\
(0.90- \\
0.99)\end{array}$ & $\begin{array}{l}1.09 \\
(0.90- \\
1.32)\end{array}$ & \\
\hline Hypertension & (Ref: no) & & $\begin{array}{l}1.01 \\
(0.98- \\
1.05)\end{array}$ & & $\begin{array}{l}1.00 \\
(0.95- \\
1.04)\end{array}$ & & $\begin{array}{l}0.90 \\
(0.75- \\
1.09)\end{array}$ & \\
\hline $\begin{array}{l}\text { Drinking } \\
\text { (Current) }\end{array}$ & (Ref: no) & & $\begin{array}{l}1.29 \\
(1.24- \\
1.35)\end{array}$ & $\begin{array}{l}0.99 \\
(0.94- \\
1.04)\end{array}$ & $\begin{array}{l}1.53 \\
(1.46- \\
1.61)\end{array}$ & $\begin{array}{l}1.16 \\
(1.09- \\
1.23)\end{array}$ & $\begin{array}{l}0.81 \\
(0.66- \\
1.01)\end{array}$ & \\
\hline $\begin{array}{l}\text { Smoking } \\
\text { (Current) }\end{array}$ & (Ref: no) & & $\begin{array}{l}1.36 \\
(1.31- \\
1.41)\end{array}$ & $\begin{array}{l}1.03 \\
(0.98- \\
1.08)\end{array}$ & $\begin{array}{l}1.42 \\
(1.36- \\
1.48)\end{array}$ & $\begin{array}{l}0.98 \\
(0.93- \\
1.04)\end{array}$ & $\begin{array}{l}1.11 \\
(0.92- \\
1.33)\end{array}$ & \\
\hline $\begin{array}{l}\text { Physical } \\
\text { activity }\end{array}$ & (Ref: no) & & $\begin{array}{l}1.02 \\
(0.98- \\
1.05)\end{array}$ & & $\begin{array}{l}1.02 \\
(0.98- \\
1.07)\end{array}$ & & $\begin{array}{l}1.17 \\
(0.97- \\
1.42)\end{array}$ & \\
\hline
\end{tabular}

Women had higher odds of dyslipidemia awareness compared with men (AOR: 1.53, 95\% Cl: 1.46-1.59). The likelihood of awareness was higher among urban compared with rural residents (AOR: 1.40, 95\% Cl: 1.35-1.45). The bivariate and multivariate analyses showed that awareness was more than three times higher in adults with a family history of dyslipidemia in their immediate family compared to those without a positive family history (AOR: 3.21, 95\% $\mathrm{Cl}$ : 2.99-3.45). Subjects with a history of CHD or stroke had positive associations with awareness [AOR (95\% CI), 
1.41 (1.33-1.49)], and [AOR (95\% Cl), 1.20 (1.15-1.25)], respectively. Participants with central obesity were more likely to be aware of dyslipidemia in both the crude and adjusted models.

Compared to men, women were more likely to receive treatment [AOR $(95 \% \mathrm{CI})], 1.78(1.69-1.88)$. The odds of treatment in individuals having a health insurance was higher than those without even after adjustment $[A O R=1.12$; $\mathrm{Cl}$ (1.05-1.19)]. Subjects with a positive personal history of $\mathrm{CHD}$ or stroke had higher adjusted odds of being treated [(AOR (95\% Cl), $1.13(1.05-1.21)]$, or [(AOR (95\% Cl), 1.23(1.17-1.30)]. Adults living in a high-income socioeconomic region were more likely to be treated for dyslipidemia. Current alcohol drinkers had higher likelihood for treatment [(AOR $(95 \% \mathrm{Cl}), 1.16(1.09-1.23)$. The probability of receiving treatment significantly decreased among adults with diabetes and those living in a stroke belt zone.

The probability of controlling dyslipidemia was higher among women [AOR (95\% CI), $1.64(1.30-2.07)$, and individuals living in northern China [AOR (95\% Cl), $1.74(1.40-2.16)]$. Table 3.

Overall, persons within the age category of 50-59 years were least likely to get their condition controlled. [OR ( $95 \%$ $\mathrm{Cl}), 0.69(0.55-0.85)]$. The likelihood of controlling dyslipidemia was $32 \%$ lower in urban than rural participants (AOR $=0.68,95 \% \mathrm{Cl}$ : $0.56-0.83)$. The probability of controlling dyslipidemia decreased with more than normal body weight (overweight and obesity), which were statistically significant. Persons with a health insurance and those living in a stroke belt zone were less likely to have controlled dyslipidemia. The bivariate analysis showed significant association between control and history of stroke [COR (95\% CI), 1.37(1.03-1.80)], and high-income region [COR $(95 \% \mathrm{Cl}), 1.66(1.29-2.12)]$, however, in the adjusted analysis, the above mentioned variables lost their significance. Table 3.

\section{Discussion}

This is a nationwide population-based report on the awareness, treatment, control and relevant determinants of dyslipidemia among middle and older aged Chinese adults. Main findings include: Sixty-four percent of subjects with dyslipidemia were aware of their condition, of whom $18.9 \%$ received pharmacological treatment, and of whom $7.3 \%$ had controlled lipids. Treatment and control proportions in both residences were higher in women than men. The following determinants showed higher likelihood for awareness: women, urban residence, junior or middle school, health insurance, family history of dyslipidemia, history of stroke, central obesity and history of CHD. Women, health insurance, history of CHD, current drinking and personal history of stroke showed positive association with treatment. Dyslipidemia control saw significant relationships with age groups, women, urban residence, northern zone, health insurance, stroke belt, overweight, and general obesity.

If our results are juxtaposed with the 'rule of halves' framework, ${ }^{10}$ obviously, the proportion of treatment (18.9\%) and control (7.3\%) observed is well below the $50 \%$ benchmark. Although, our result suggests that awareness has improved, more actions are needed to scale-up and improve treatment of lipid disorders in China. The present results on awareness, treatment and control were higher compared with previous studies in China ${ }^{9,22}$ Further, we recorded higher awareness and treatment, but lower control values for dyslipidemia compared with other studies. ${ }^{7,8}$ The low level of control observed in the current study could partly be explained by the low treatment value observed despite

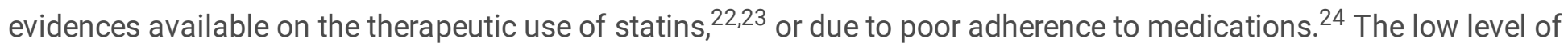
control seen may also reveal some level of ineffectiveness in the current treatment approaches. Therefore, strategies to improve patient's adherence to medication regimens, the increased use of treatment protocols that ensure adequate treatment and access to more affordable medications should be targeted. Notably, drugs therapy alone does not control dyslipidemia completely. Measures such as lifestyle modifications and good nutrition are effective 
in controlling high blood lipids. ${ }^{25-27}$ There is also the need to place more emphasis on prevention. Efforts are needed to scale-up both targeted and opportunistic screening programs, health promotions, and provision of more effective cholesterol-modifying medications for use. Early identification of populations who are not only at risk, but who could benefit most from preventive measures is crucial. Hence, the use of both pharmacological and non- pharmacological approaches are preferred for effective dyslipidemia management. The low treatment and control identified present a great opportunity for China to improve its dyslipidemia management strategy.

In the present study, subjects aged 40-49 years had higher odds for dyslipidemia control than older participants. This results was in accord to data from a Korean study where younger adults were more likely to have controlled LDL$\mathrm{C}$ than the elderly. ${ }^{28}$ However, many reverse results have been reported between older ages and dyslipidemia control. $7,22,29$ Again, differing from our findings, a positive association between aging and the control of chronic diseases such as hypertension has been reported. ${ }^{30}$ Our results may support the finding that dyslipidemias such as raised TC control rates may differ depending on the participants and country, and rates may range from $18-100 \%{ }^{31}$

Consistent with prior reports, ${ }^{22,29}$ we showed that women were more likely than men to have dyslipidemia awareness, treatment and control. Similarly, two studies on the management of raised LDL-C indicated that women recorded higher likelihood of awareness, treatment and control. 6,32 Behavioral differences between the sexes could partly explain this results, as women are reported to seek healthcare services more often than men. ${ }^{6}$ An earlier study 18 reported no relationship between dyslipidemia treatment and place of residence, but our study demonstrated positive association between dyslipidemia awareness or treatment with urban residence. Similarly, studies in Malaysia and Thailand, ${ }^{33,34}$ showed high awareness and treatment levels between raised LDL-C and urban centers. Further, findings from studies in low and middle-income countries have reported lower values of dyslipidemia awareness and treatment in rural settings. ${ }^{19,35}$ The high awareness and treatment levels found in urban areas may be attributed to wealthier and better educated populations usually found in cities. ${ }^{36}$ Again, it may buttress the evidence of difficult access to health care common in rural areas. ${ }^{11}$ Hence, health promotion programs should target areas such as rural settings with limited healthcare resources. The lower level of control seen among urban populations merits some explanation, albeit difficult, as poor adherence to treatment regimens may play a role. Junior/middle school education level was independently related to dyslipidemia awareness, but not treatment and control. This result contradicts previous reports. ${ }^{7,18}$ The mechanisms underlying the link between length of education and awareness of dyslipidemia is not clear. Nevertheless, a previous report suggested that education is the finest socioeconomic status index and can predict CVD's awareness. ${ }^{37}$ Future studies are warranted to examine this results.

Health insurance coverage was associated with dyslipidemia awareness and treatment, but not control. In line with our finding, a US study found respondents without health insurance with lower levels of awareness and treatment for elevated LDL-C. ${ }^{38}$ Insured individuals were more likely to receive treatment in another study. ${ }^{38}$ Once dyslipidemia is seen, effective management follows, and health insurance fundamentally addresses socioeconomic barriers to effective healthcare. ${ }^{38}$ The negative relationship that existed between having health insurance and dyslipidemia control in the current study was inconsistent with earlier reports, ${ }^{6,38,39}$ since health insurance is related to improved CV risk factor control and outcomes. ${ }^{39}$

Residing in a high compared with low-income region was associated with less dyslipidemia awareness, but high treatment level. This may suggest that high-income region is an independent driver for treatment. High- income communities have been reported to demonstrate high association with treatment of chronic conditions. ${ }^{40}$ It may be 
that, the availability or easier access to health facilities in high-income regions facilitates the usage of health services.

From this study, staying in northern China was an independent determinant for dyslipidemia control. A study determined that stroke prevalence was significantly higher in the northern parts of China, ${ }^{41}$ and living in the north was associated with high dyslipidemia prevalence. ${ }^{8,42}$ We speculate that, the high prevalence of stroke and dyslipidemia found in the north could positively affect inhabitants' behaviors towards treatment and yield favorable results. We showed that lower likelihoods of dyslipidemia awareness, treatment, and control were related to living in the stroke belt region of China. This results was dissimilar to that of the REGARDS study ${ }^{43}$ where lower control rate was found outside the stroke belt region. This finding may indicate that effective and efficient management of lipid disorders may not be related to the stroke belt zone. Studies are required to further investigate why awareness and treatment of dyslipidemia were less likely in the stroke belt zone. We observed a negative association between dyslipidemia awareness and obesity. This was consistent with an earlier study. ${ }^{44}$ Speculatively, the low awareness level seen could be due to the natural history of the disease, or one's poor attitude towards healthcare. Overweight and general obesity were significant negative predictor for dyslipidemia control, these were similar to earlier researches. ${ }^{7,18}$ An explanation could be that dyslipidemia control is difficult in individuals with more than normal body weight. Central obesity was a significant positive predictor of dyslipidemia awareness. This finding might reflect efforts instituted by healthcare systems to improve screening for blood lipid disorders among high-risk individuals. ${ }^{45}$

Subjects with a personal history of CHD or stroke were more likely to know or receive treatment for dyslipidemia. Similarly, a previous study observed that personal history of ASCVDs were associated with higher use of statins. ${ }^{46}$ In addition, other reports [35-37] had observed that people who experienced cardiovascular events often become more focused on their health, especially on CVD risk factors such as dyslipidemia. Therefore, these individuals are more likely to comply with lipid-modifying medications and/ or lifestyle interventions.

Patients with family history of dyslipidemia in their immediate family had the strongest independent association of awareness in this study. This is in line with the result of He et al. where persons with a family history of dyslipidemia had higher likelihood of awareness. ${ }^{7}$ It has also been found that the chances of diagnosis and treatment of dyslipidemia is generally higher in high-risk individuals including those with CVDs. Predictably, family members would be more mindfulness and watchfulness of dyslipidemia and doctors would pay more attention to these patients. $^{7}$

Subjects with diabetes were less likely to get treatment for dyslipidemia. A reverse results was seen by Li et al. ${ }^{41}$ where dyslipidemia treatment among persons with diabetes was up to about $55.9 \%$. We speculate that there persons did not receive adequate treatment due to wrong attitude towards the disease or poor adherence to treatment. ${ }^{47,48}$. The low likelihood of treatment could also be as a result poor knowledge about diabetic dyslipidemia among patients. Therefore, it is imperative for health workers to intensity health education and pay more attention to detect lipid disorders in suspected co-morbid patients. This study found that current drinking was associated with dyslipidemia treatment. This was dissimilar to findings in the Jilin province of China, ${ }^{7}$ where current drinking was associated with low level of treatment. We speculate that these individuals were already in the health service system, so they were more likely to be treated with medication.

\section{Strengths And Limitations}


Key strengths of the study include its population based design, representation of a wide range of urban and rural communities across China. Thus, this nationwide coverage allows for calculation of nationally representative estimates. Further, these analyses consisted of several determinants of dyslipidemia awareness, treatment, and control, which may provide a broader view of the relative role of different determinants in prevention efforts.

This survey was cross-sectional, thus, exploring casualty was limited. Another limitation observed was that participants' previous experience on medication use for other conditions could affect treatment adherence. Also, we did not investigate the effect of cognitive factors such as self-efficacy and illness perception of people who received treatment and had controlled dyslipidemia. Information bias might occur from self-reported characteristics.

\section{Conclusion}

In summary, dyslipidemia awareness was high (64.0\%), with low treatment (18.9) and control (7.3) proportions. Higher proportions of awareness, treatment and control were seen in women than men. Treatment was higher in rural than urban men (15.4\% vs. $10.6 \%)$, but women in urban areas showed higher proportion for treatment versus their rural counterparts (26.9\% vs. $20.8 \%$ ). Dyslipidemia control was more in women than men in both rural and urban areas.

Women, urban residence, family history of dyslipidemia and central obesity were positive associated factors of awareness. Personal history of stroke or CHD, having health insurance, alcohol drinkers and women were more likely to be treated for dyslipidemia. Diabetics and living in a stroke belt region had significant negative association with treatment. Regarding dyslipidemia control, health insurance, general and central obesity recorded negative relationship with control. Therefore, improved health education and treatment are needed for better dyslipidemia management.

\section{Abbreviations}

CNSSPP: China National Stroke Screening and Prevention Project CVD:Cardiovascular disease DALYs:disabilityadjusted life-years TC:Raised total cholesterol TG:Raised triglycerides LDL-C:Raised low-density lipoprotein HDLC:Low high-density lipoprotein ASCVD:Atherosclerotic cardiovascular disease

\section{Declarations}

\section{Acknowledgements}

The authors are grateful to all study participants of the CNSSPP, faculty and colleagues for their contributions in this study.

\section{Funding}

This study was funded by the Ministry of Finance of the People's Republic of China (Finance and Social Security [2011] Document No. 61, Ministry of Finance). Innovation Committee of Shenzhen Science and Technology (KJYY20170413162318686) funded ZHW. ZXL was funded by the Fundamental Research Funds for the Central Universities, Huazhong University of Science and Technology, Wuhan, China (2016YXMS215). YG was funded by the China Postdoctoral Science Foundation funded project (2018M630870).

\section{Availability of data}

Page $17 / 22$ 
The dataset of the study is available from the corresponding author on reasonable request.

\section{Authors' contributions}

SO developed the research question and designed, and conducted analysis of the manuscript, SO, YG, EAY and DT critically reviewed the manuscript and ZXL approved the final design and provided supervision. All authors have approved the final manuscript.

\section{Ethics approval}

This study was approved by the Ethics Committee of the Xuanwu Hospital Institutional Review Board, Capital Medical University (Beijing, China), and performed according to the declaration of Helsinki.

\section{Consent for publication}

Not applicable.

\section{Competing interests:}

None

\section{References}

1. McQueen MJ, Hawken S, Wang X, et al. Lipids, lipoproteins, and apolipoproteins as risk markers of myocardial infarction in 52 countries (the INTERHEART study): a case-control study. Lancet (London England). 2008;372(9634):224-33.

2. Rosamond W, Flegal K, Friday G, et al. Heart disease and stroke statistics-2007 update: a report from the American Heart Association Statistics Committee and Stroke Statistics Subcommittee. Circulation. 2007;115(5):e69-171.

3. Erqou S, Kaptoge S, Perry PL, et al. Lipoprotein(a) concentration and the risk of coronary heart disease, stroke, and nonvascular mortality. Jama. 2009;302(4):412-23.

4. Kuklina EV, Yoon PW, Keenan NL. Trends in high levels of low-density lipoprotein cholesterol in the United States, 1999-2006. Jama. 2009;302(19):2104-10.

5. Farzadfar F, Finucane MM, Danaei G, et al. National, regional, and global trends in serum total cholesterol since 1980: systematic analysis of health examination surveys and epidemiological studies with 321 country-years and 3.0 million participants. Lancet (London England). 2011;377(9765):578-86.

6. Goff DC Jr, Bertoni AG, Kramer H, et al. Dyslipidemia prevalence, treatment, and control in the Multi-Ethnic Study of Atherosclerosis (MESA): gender, ethnicity, and coronary artery calcium. Circulation. 2006;113(5):647-56.

7. He H, Yu YQ, Li Y, et al. Dyslipidemia awareness, treatment, control and influence factors among adults in the Jilin province in China: a cross-sectional study. Lipids Health Dis. 2014;13:122.

8. Song $\mathrm{P}$, Zha M, Yang $\mathrm{X}$, et al. Socioeconomic and geographic variations in the prevalence, awareness, treatment and control of dyslipidemia in middle-aged and older Chinese. Atherosclerosis. 2019;282:57-66.

9. Huang Y, Gao L, Xie X, Tan SC. Epidemiology of dyslipidemia in Chinese adults: meta-analysis of prevalence, awareness, treatment, and control. Popul Health Metr. 2014;12(1):28.

10. Hart JT. Rule of halves: implications of increasing diagnosis and reducing dropout for future workload and prescribing costs in primary care. Br J Gen Pract. 1992;42(356):116-9. 
11. Janus ED, Tideman PA, Dunbar JA, et al. Dyslipidaemia in rural Australia: prevalence, awareness, and adherence to treatment guidelines in the Greater Green Triangle Risk Factor Study. Med J Aust. 2010;192(3):127-32.

12. Wang X, Li W, Song F, et al. Carotid Atherosclerosis Detected by Ultrasonography: A National Cross-Sectional Study. J Am Heart Assoc. 2018;7(8).

13. Fu W, Cao S, Liu B, et al. Association of general and central adiposity with blood pressure among Chinese adults: results from the China National Stroke Prevention Project. Journal of hypertension. 2018;36(12):2406-13.

14. Yan S, Li J, Li S, et al. The expanding burden of cardiometabolic risk in China: the China Health and Nutrition Survey. Obes Rev. 2012;13(9):810-21.

15. Jones-Smith JC, Popkin BM. Understanding community context and adult health changes in China: development of an urbanicity scale. Soc Sci Med. 2010;71(8):1436-46.

16. Gan Y, Wu J, Zhang S, et al. Prevalence and risk factors associated with stroke in middle-aged and older Chinese: A community-based cross-sectional study. Sci Rep. 2017;7(1):9501.

17. Opoku S, Gan Y, Fu W, et al. Prevalence and risk factors for dyslipidemia among adults in rural and urban China: findings from the China National Stroke Screening and prevention project (CNSSPP). BMC Public Health. 2019;19(1):1500.

18. Zhang FL, Xing YQ, Wu YH, et al. The prevalence, awareness, treatment, and control of dyslipidemia in northeast China: a population-based cross-sectional survey. Lipids Health Dis. 2017;16(1):61.

19. Pan L, Yang Z, Wu Y, et al. The prevalence, awareness, treatment and control of dyslipidemia among adults in China. Atherosclerosis. 2016;248:2-9.

20. Chobanian AV, Bakris GL, Black HR, et al. Seventh report of the Joint National Committee on Prevention, Detection, Evaluation, and Treatment of High Blood Pressure. Hypertension (Dallas Tex: 1979). 2003;42(6):1206-52.

21. Joint committee for guideline r. 2016 Chinese guidelines for the management of dyslipidemia in adults. J Geriatr Cardiol. 2018;15(1):1-29.

22. Cai L, Zhang L, Liu A, Li S, Wang P. Prevalence, awareness, treatment, and control of dyslipidemia among adults in Beijing, China. J Atheroscler Thromb. 2012;19(2):159-68.

23. Gould AL, Davies GM, Alemao E, Yin DD, Cook JR. Cholesterol reduction yields clinical benefits: meta-analysis including recent trials. Clin Ther. 2007;29(5):778-94.

24. Brown MT, Bussell JK. Medication adherence: WHO cares? Mayo Clin Proc. 2011;86(4):304-314.

25. Yoon SS, Carroll MD, Johnson CL, Gu Q. Cholesterol management in the United States: the national health and nutrition examination survey, 1999 to 2006. Ann Epidemiol. 2011;21(5):318-26.

26. Arao T, Oida Y, Maruyama C, et al. Impact of lifestyle intervention on physical activity and diet of Japanese workers. Prev Med. 2007;45(2-3):146-52.

27. Moran A, Gu D, Zhao D, et al. Future cardiovascular disease in china: markov model and risk factor scenario projections from the coronary heart disease policy model-china. Circ Cardiovasc Qual Outcomes. 2010;3(3):24352.

28. Lee YH, Lee SG, Lee MH, et al. Serum cholesterol concentration and prevalence, awareness, treatment, and control of high low-density lipoprotein cholesterol in the Korea National Health and Nutrition Examination Surveys 2008-2010: Beyond the Tip of the lceberg. J Am Heart Assoc. 2014;3(1):e000650.

29. Liu X, Yu S, Mao Z, et al. Dyslipidemia prevalence, awareness, treatment, control, and risk factors in Chinese rural population: the Henan rural cohort study. Lipids Health Dis. 2018;17(1):119. 
30. Gu D, Reynolds K, Wu X, et al. Prevalence, Awareness, Treatment, and Control of Hypertension in China. Hypertension (Dallas Tex: 1979). 2002;40(6):920-7.

31. Tolonen H, Keil U, Ferrario M, Evans A. Prevalence, awareness and treatment of hypercholesterolaemia in 32 populations: results from the WHO MONICA Project. Int J Epidemiol. 2005;34(1):181-92.

32. Lotufo PA, Santos RD, Figueiredo RM, et al. Prevalence, awareness, treatment, and control of high low-density lipoprotein cholesterol in Brazil: Baseline of the Brazilian Longitudinal Study of Adult Health (ELSA-Brasil). J Clin Lipidol. 2016;10(3):568-76.

33. Aekplakorn W, Taneepanichskul S, Kessomboon P, et al. Prevalence of Dyslipidemia and Management in the Thai Population, National Health Examination Survey IV, 2009. J Lipids. 2014;2014:249584-4.

34. Ab Majid NL, Omar MA, Khoo YY, et al. Prevalence, Awareness, Treatment and Control of hypertension in the Malaysian population: findings from the National Health and Morbidity Survey 2006-2015. Journal of human hypertension. 2018;32(8-9):617-24.

35. Joshi SR, Anjana RM, Deepa M, et al. Prevalence of dyslipidemia in urban and rural India: the ICMR-INDIAB study. PLoS One. 2014;9(5):e96808.

36. Supiyev A, Nurgozhin T, Zhumadilov Z, Peasey A, Hubacek JA, Bobak M. Prevalence, awareness, treatment and control of dyslipidemia in older persons in urban and rural population in the Astana region, Kazakhstan. BMC Public Health. 2017;17(1):651.

37. Winkleby MA, Jatulis DE, Frank E, Fortmann SP. Socioeconomic status and health: how education, income, and occupation contribute to risk factors for cardiovascular disease. Am J Public Health. 1992;82(6):816-20.

38. Egan BM, Li J, Sarasua SM, et al. Cholesterol Control Among Uninsured Adults Did Not Improve From 20012004 to 2009-2012 as Disparities With Both Publicly and Privately Insured Adults Doubled. J Am Heart Assoc. 2017;6(11).

39. Fowler-Brown A, Corbie-Smith G, Garrett J, Lurie N. Risk of cardiovascular events and death-does insurance matter? Journal of general internal medicine. 2007;22(4):502-7.

40. Chow CK, Teo KK, Rangarajan S, et al. Prevalence, awareness, treatment, and control of hypertension in rural and urban communities in high-, middle-, and low-income countries. Jama. 2013;310(9):959-68.

41. Li Q, Wu H, Yue W, et al. Prevalence of Stroke and Vascular Risk Factors in China: a Nationwide Communitybased Study. Scientific Reports. 2017;7(1):6402.

42. Gu D, Gupta A, Muntner P, et al. Prevalence of cardiovascular disease risk factor clustering among the adult population of China: results from the International Collaborative Study of Cardiovascular Disease in Asia (InterAsia). Circulation. 2005;112(5):658-65.

43. Zweifler RM, McClure LA, Howard VJ, et al. Racial and geographic differences in prevalence, awareness, treatment and control of dyslipidemia: the reasons for geographic and racial differences in stroke (REGARDS) study. Neuroepidemiology. 2011;37(1):39-44.

44. Reiger S, Jardim TV, Abrahams-Gessel S, et al. Awareness, treatment, and control of dyslipidemia in rural South Africa: The HAALSI (Health and Aging in Africa: A Longitudinal Study of an INDEPTH Community in South Africa) study. PLoS One. 2017;12(10):e0187347.

45. Roth GA, Fihn SD, Mokdad AH, Aekplakorn W, Hasegawa T, Lim SS. High total serum cholesterol, medication coverage and therapeutic control: an analysis of national health examination survey data from eight countries. Bull World Health Organ. 2011;89(2):92-101.

46. Bucholz EM, Rodday AM, Kolor K, Khoury MJ, de Ferranti SD. Prevalence and Predictors of Cholesterol Screening, Awareness, and Statin Treatment Among US Adults With Familial Hypercholesterolemia or Other Forms of 
Severe Dyslipidemia (1999-2014). Circulation. 2018;137(21):2218-30.

47. Colhoun HM, Betteridge DJ, Durrington PN, et al. Primary prevention of cardiovascular disease with atorvastatin in type 2 diabetes in the Collaborative Atorvastatin Diabetes Study (CARDS): multicentre randomised placebocontrolled trial. The Lancet. 2004;364(9435):685-96.

48. Collins R, Armitage J, Parish S, Sleight P, Peto R. Effects of cholesterol-lowering with simvastatin on stroke and other major vascular events in 20536 people with cerebrovascular disease or other high-risk conditions. Lancet (London England). 2004;363(9411):757-67.

\section{Figures}

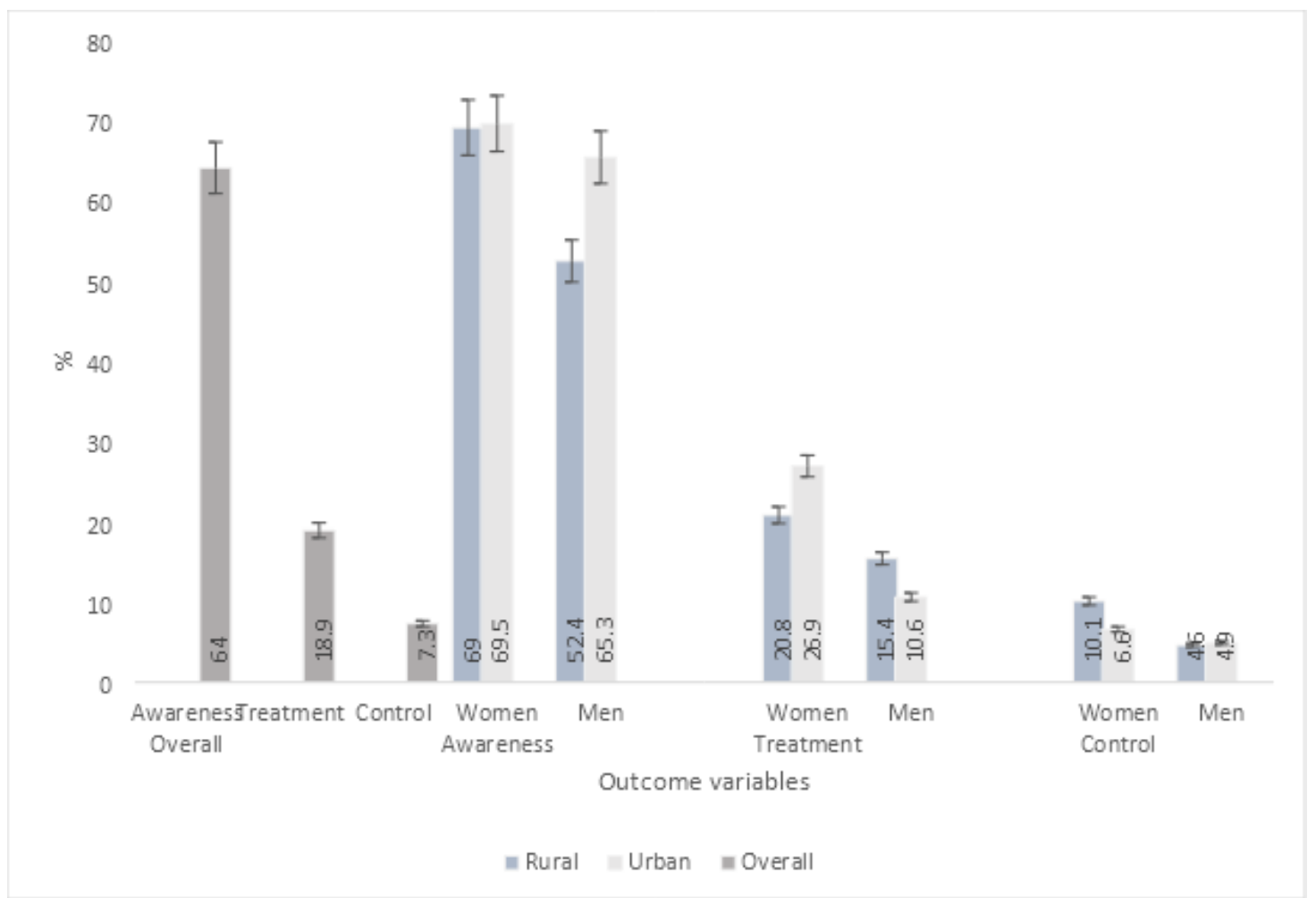

\section{Figure 1}

The overall, sex and rural/urban proportions of dyslipidemia awareness, treatment and control among the study population. 


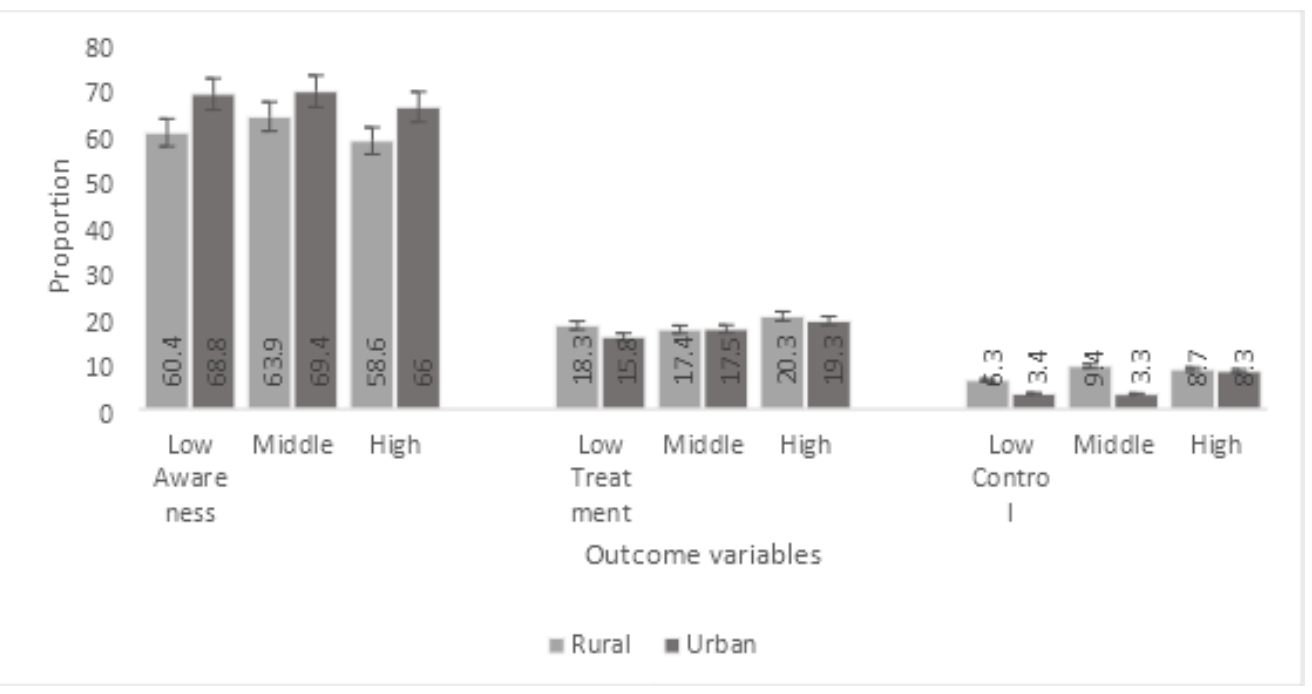

\section{Figure 2}

Proportions of dyslipidemia awareness, treatment and control among rural and urban populations compared with the socioeconomic regions (low, middle, high) of China

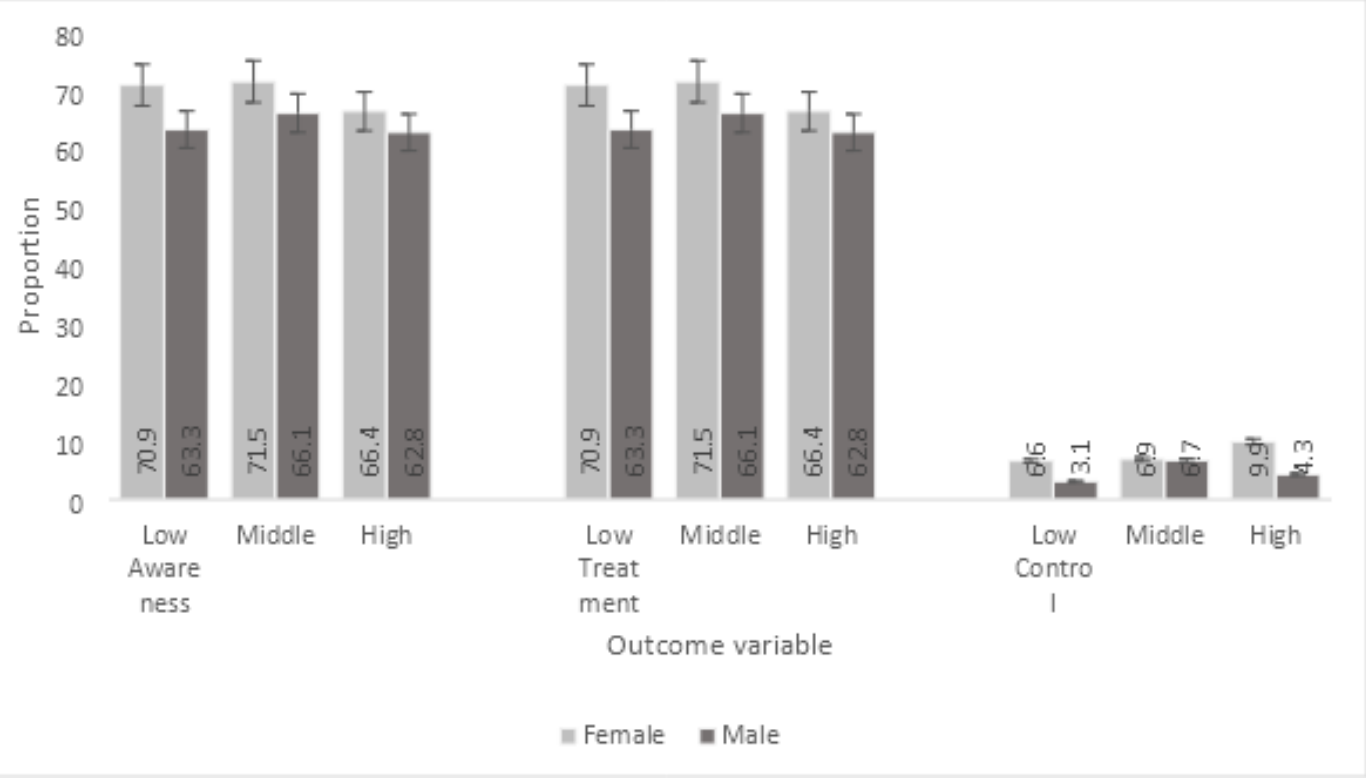

\section{Figure 3}

Proportions of dyslipidemia awareness, treatment and control among men and women compared with socioeconomic regions (low, middle, high) of China.

\section{Supplementary Files}

This is a list of supplementary files associated with this preprint. Click to download.

- CoverLetter.docx 